Objectives: The aim of this study was to present an overview of the evidence of the effect of job loss prevention interventions, which can improve work participation and decrease absenteeism and job loss in persons with IA

Methods: A systematic literature search was performed in the databases PubMed, EMBASE, CINAHL, PsycINFO and the Cochrane Library in two steps: 1) an update of the Cochrane review, restricted to studies published from January 2014 to February 2019 and 2) an additional search with updated keywords with no time restriction. Quality assessment and data extraction were performed independently by two authors. The results were summarized narratively.

Results: The first search identified 1276 titles and the second search identified 2384 titles. Six studies (including the three RCT's included in the Cochrane review (1)) were included. The results indicated that job loss prevention interventions may have effect on work ability, absenteeism and in particular job loss, but the results across study outcomes, were not consistent. This may be due to heterogeneity in the interventions delivered (i.e. dose, duration and setting) and outcome measures used. Most of the studies were of low quality. Therefore, the results should be interpreted with caution.

Conclusion: Job loss prevention interventions may have an effect on work ability, absenteeism and in particular job loss among persons with IA. Further studies of high quality regarding job loss prevention interventions for people with IA are recommended.

References:

[1] Hoving, J.L. et al. 2014, "Non-pharmacological interventions for preventing job loss in workers with inflammatory arthritis", The Cochrane database of systematic reviews, vol. 11;2014; , no. 11, pp. CD010208.

Disclosure of Interests: Christina Merete Tvede Madsen: None declared, Sara Kjaer: None declared, Jette Primdahl Speakers bureau: BMS and Pfizer, Jeanette Reffstrup Christensen: None declared, Cecilie von Bülow: None declared DOI: 10.1136/annrheumdis-2020-eular.1309

\section{FRI0625-HPR APPS TARGETING SYMPTOMS ASSOCIATED WITH SJÖGREN'S SYNDROME AND POTENTIAL USERS' PERCEPTIONS OF THEIR FEATURES: CONTENT ANALYSIS AND THINK ALOUD STUDY}

C. Mccallum ${ }^{1}$, M. Campbell ${ }^{2}$, M. Higgs ${ }^{1}$, J. Vines ${ }^{1}$, T. Rapley ${ }^{1}$, K. Hackett ${ }^{1}$ ${ }^{1}$ Northumbria University, Newcastle upon Tyne, United Kingdom; ${ }^{2}$ Teesside University, Middlesbrough, United Kingdom

Background: Sjögren's syndrome (SS) is a rheumatic disease requiring self-management which may be delivered through smartphones. When developing digital interventions it is important to review what is already available (market segmentation $)^{1}$ to identify unique selling points and aid uptake and adoption. While there are no dedicated SS apps, many are publicly available for other rheumatic conditions $^{2}$. Understanding user preferences for existing apps may help to design an engaging app for SS self-management.

Objectives: To explore apps targeting SS symptoms of dryness, sleep disturbances, fatigue and pain. To explore views of people with SS on these app features.

Methods: Apple Store apps were retrieved on 04 March 2019 using the following search terms: dry, dry eye, sleep, insomnia, fatigue, tiredness and pain. Included apps were English and in Medical or Health \& Fitness genres. Exclusion criteria were; duplicates, additional external devices required and apps targeting alcohol reduction or children

Included apps were grouped by symptom. App descriptions were open-coded to generate a thematic coding framework (i.e. full list of features) for each symptom which was then applied to all app descriptions. To obtain views of people with SS, several of the reviewed apps for each symptom covering the full list of features were given to 13 focus group participants to use in 'think aloud' sessions $(n=4)$. Audio data was recorded, transcribed and deductively analysed using the framework to gather opinions relating to each feature.

Results: Of 914 apps retrieved, 542 were included. Features within apps targeting dryness $(n=15)$ provided dry eye information, self-assessment and reminders to blink or look away from screens. Apps targeting sleep $(n=310)$ included features to support sleep restriction, sleep hygiene, sleep tracking (sleep onset and wake up times, time in bed, overall sleep quality), relaxing sounds, guided meditation, sleep stories, snore recording and alarms. Fatigue apps $(n=79)$ included features to detect current physical and mental fatigue levels, support pacing (i.e. track fatigue, label tasks as 'high energy', prioritise tasks), and self-massage instructions. Apps targeting pain $(n=138)$ featured pain tracking (of severity, affected body areas), guided exercises, and mindfulness.

Dryness apps prompted participants to reflect on its impact on daily activities, but further dryness features were desired relating to: using a humidifier; eye drop reminders; and dryness tips for other body areas e.g. vaginal dryness. Sleep restriction features were believed to be irrelevant but viewing and selecting sleep hygiene tips to "try" were considered useful. Beyond entering sleep onset and wake up times, participants wished to track "when and why I woke up", to understand night awakenings in relation to other symptoms. Fatigue detection features were felt to be more useful for those recently diagnosed, as experienced participants could easy identify when they were fatigued ("I don't need an app to tell me!"). Participants valued pacing features but found them difficult to use. Daily pain tracking was considered demotivating, but useful for remembering and explaining issues to healthcare professionals. Participants believed that a dedicated app for SS would support self-management and raise SS awareness.

Conclusion: Existing apps targeting SS symptoms do not meet the needs of those with SS. App features should be tailored to SS by supporting dryness management in body areas beyond eyes, and night-awakenings. Pacing features must be easy to use. The ability to track pain should be optional and tracking prompts should be limited. Design considerations should be implemented alongside evidence-based behaviour change techniques to support self-management. References:

[1] Araújo-Soares, V. et al (2019). European Psychologist, 24(1), 7

[2] Knitza, J., et al (2019). JMIR mHealth and uHealth, 7(8), e14991

Acknowledgments: Versus Arthritis (Grant 22026)

Disclosure of Interests: None declared

DOI: 10.1136/annrheumdis-2020-eular.997

\section{FRI0626-HPR DEVELOPMENT OF A TOOL TO SUPPORT PATIENT AND NURSE IN COMMUNICATING ON SELF- MANAGEMENT SUPPORT NEEDS PROJECT OF V\&VN RHEUMATOLOGY RESEARCH GROUP}

A. J. L. Meier ${ }^{1}$, Y. Van Eijk-Hustings ${ }^{2}$, B. Maat ${ }^{3} .{ }^{1}$ Máxima MC, Department of Rheumatology, Regional Rheumatology Center, Eindhoven, Netherlands; ${ }^{2}$ Maastricht University Medical Centre, Department of Epidemiology and Medical Technology Assessment, Maastricht, Netherlands; ${ }^{3}$ ReumaZorg Nederland, Patient Research Partner, Nijmegen, Netherlands

Background: Self-management, ability of patients to optimally integrate their illness or disorder into their daily lives, is explicitly part of the new definition of health.

Self-management is considered essential in rheumatology care but is also a challenge for patients and rheumatology nurses. Often patients need support but clarifying their support needs is difficult. Also, nurses experience difficulties in providing self-management support. A communication tool might help patients and nurses.

Objectives: In preparation for development of an online self-management program, a framework comprising 55 needs from 11 different domains has been developed (1).

The objective of this study is to develop a tool, based on this existing framework that can support patients and nurses in making targeted choices for adequate support.

Methods: The tool was developed in two steps. First, it was explored if existing framework as such was useful as a basis for the tool. It was discussed in a brainstorming session with rheumatology patients and rheumatology nurses. Second, two additional workshops, one with patients and one with nurses were organised. In these sessions, yellow cards with 55 needs from the framework (1) were compared with a more generic framework for self-management support in chronic diseases: the self-management web (2), figure 1. Goal of the workshop was to gain insight into usability of models and to gauge the ideas for a tool. The choice for these two models was based on the scientific background as well as the use with patients with a chronic or rheumatic disease.



Picture 1 : Workshop

Results: In the first brainstorming session 5 patients and 5 rheumatology nurses participated. 